\title{
Characterization of ORF127 of Helicoverpa armigera nucleopolyhedrovirus
}

\author{
Z.J. GUO' ${ }^{1}$ L.H. QIU' ${ }^{2}$, M.F. DU³, S.H. AN ${ }^{3}$ \\ ${ }^{1}$ Institute of Life Science, ${ }^{2}$ School of Finance and Economics, Jiangsu University, 301 Xuefu Road, Zhenjiang 212013, Jiangsu, P.R. \\ China; ${ }^{3}$ College of Plant Protection, Henan Agricultural University, 95 Wenhua Road, Zhengzhou 450002, Henan, P.R. China
}

Received April 17, 2009; accepted September 21, 2009

\begin{abstract}
Summary. - It has been shown that Ha127 in the genome of Helicoverpa armigera nucleopolyhedrovirus (HaNPV) has homologs in some other baculoviruses and encodes a putative protein of 192 aa. In this study, a sequence analysis showed the transcription initiation site in Ha127 gene at nts 188 upstream of the translation initiation codon ATG and a potential leucine zipper motif at aa 34-55 in the corresponding protein. Ha127 transcripts were detected in HaNPV-infected HzAM1 cells at 18-72 hrs post infection ( p.i.) by RT-PCR, while the corresponding protein was found at 24-72 hrs p.i. by Western blot analysis suggesting that Ha127 is a late gene product. The size of detected Ha127 protein was about $28 \mathrm{~K}$, a larger value than the predicted $22.6 \mathrm{~K}$ indicating a post-translational modification. Immunofluorescence assay of HzAM1 cells infected with HaNPV and Ha127EGFP expression showed that Ha127 protein was localized in the nucleus. In summary, these data suggested that Ha127 was a functional ORF that might play a role in the nucleus during the late or very late gene expression.
\end{abstract}

Keywords: Helicoverpa armigera nucleopolyhedrovirus; ORF127; transcription; translation; sub-cellular localization

\section{Introduction}

Baculoviridae is the family of DNA viruses that contain circular, supercoiled double-stranded DNA within a rod-shaped nucleocapsid. This virus family has two genera Nucleopolyhedrovirus and Granulovirus (Theilmann et al., 2005), but their reclassification was proposed (Jehle et al., 2006). At present, according to the virus taxonomy list on ICTV website, this family has four genera Alphabaculovirus, Betabaculovirus, Deltabaculovirus, and Gammabaculovirus.

E-mail: gzh762677@ujs.edu.cn; fax: +86-511-88791923.

Abbreviations: 5'-RACE $=5$ ' rapid amplification of cDNA ends; BV = budded virus; ChchNPV $=$ Chrysodeixis chalcites NPV; ClbiNPV = Clanis bilineata NPV; EGFP = enhanced green fluorescence protein; Ha127 = ORF127 gene or protein; HaMNPV = Helicoverpa armigera multiple NPV; HaNPV = Helicoverpa armigera NPV; HzSNPV = Helicoverpa zea single NPV; LdMNPV = Lymantria dispar multiple NPV; MacoNPV(A) = Mamestra configurata NPV (A); MacoNPV(B) = Mamestra configurata NPV (B); OrleNPV = Orgyia leucostigma NPV; p.i. = post infection; SeMNPV = Spodoptera exigua multiple NPV; SpliNPV = Spodoptera littoralis NPV; SpltNPV = Spodoptera litura NPV; TnSNPV = Trichoplusia ni single NPV
As a member of the genus Alphabaculovirus, HaNPV is a selective, highly infectious pathogen to the cotton bollworm Helicoverpa armigera, an insect pest causing serious damage to a variety of agricultural crops such as cotton, tobacco, pepper, tomato, maize, sorghum, and soybean (King, 1994). Since the first isolation of this pathogen in Hubei province, P.R. China, HaNPV has been extensively used for the control of $H$. armigera (Wang et al., 2004). To date, the genomes of two HaNPV isolates, i.e. HaNPV G4 with a genome of $131.4 \mathrm{~kb}$ (Chen et al., 2001) and HaNPV C1 with a genome of $130.5 \mathrm{~kb}$ (Zhang et al., 2005) were completely sequenced. The functions of individual genes such as Ha107 (Pan et al., 2007), pifs (Song et al., 2008), Ha2 (Wang et al., 2007, 2008), Ha133 (Long et al., 2006), and fp25k (Wu et al., 2005) were well-studied. Ha127 gene of HaNPV encodes a protein of 192 amino acids with a predicted Mr of $22.6 \mathrm{~K}$. The sequence of this ORF is uncommon and corresponding homologies were found only in some members of the genus Alphabaculovirus suggesting that this gene may play a specific role in the virus life cycle, but no specific function has been reported so far.

In this study, the Ha127 gene of HaNPV was characterized regarding to its transcription and expression. In addition, 
the sub-cellular localization of the corresponding protein was demonstrated.

\section{Materials and Methods}

Virus, H. armigera, cells. A culture of $H$. armigera larvae was maintained on the artificial diet at $27 \pm 1^{\circ} \mathrm{C}, 16: 8 \mathrm{hrs}$ light/dark photoperiod, and $75 \%$ relative humidity. The wild-type HaNPV G4 was propagated in the larvae and Helicoverpa zea cell line, HzAM1. This cell line was cultured at $27^{\circ} \mathrm{C}$ in TNM-FH medium (Sigma) supplemented with $10 \%$ fetal bovine serum (Gibco-BRL).

Construction of a plasmid expressing GST-Ha127 fusion protein and preparation of an antiserum. The expression of Ha127 protein was performed as described by Guo et al. (2005). Briefly, the complete Ha127 gene was amplified from HaNPV genome by PCR with primers 127-F: AGGATCCATGCATCG GTCCAATAG TAA (BamHI site underlined) and 127-R: ACTCGAGTCAATGCT CAATCGTGGCGA (XhoI site underlined). The PCR products were ligated into pGEM $^{\circledR}$-T Easy vector (Promega) and subcloned into BamHI/XhoI sites of pGEX-4T-2 (Amersham Pharmacia Biotech). The produced recombinant plasmid, pGEX-GST-Ha127 was transformed into Escherichia coli BL21 (DE3) LysS cells. GST-fused protein, GST-Ha127, was induced by $0.1 \mathrm{mmo} / 1$ isopropyl $\beta$-D-thiogalactoside (Sigma), and purified according to the method described in the GST Gene Fusion System Manual (Amersham Pharmacia Biotech). The polyclonal Ha127 antiserum was prepared according to the procedure described previously (Wang et al., 2005).

RT-PCR for Ha127 transcripts. HzAM1 cells were infected with HaNPV BVs at 5 TCID $_{50} /$ cell. Total RNA was extracted at $0,3,6,12,18,24,48$, and 72 hrs p.i. by adding RNAiso Reagent (TaKaRa Bio). The RNA was dissolved in $25 \mu$ of double-distilled water, quantified by $\mathrm{A}_{260}$ measurement, and treated with RNasefree DNase to eliminate any potential HaNPV genomic DNA contamination. First strand cDNA was synthesized with AMV reverse transcriptase XL (TaKaRa Bio) and oligo(dT) primer. The obtained cDNA mixtures were amplified by PCR using primers $127-\mathrm{F}$ and 127-R. RNA from mock-infected cells was used as a control.

5'-RACE analysis. The 5'-end of Ha127 transcript was determined according to the manufacturer's method supplied with 5'-Full RACE Kit (TaKaRa Bio), using $2 \mu \mathrm{g}$ of total RNA obtained at 18 and $48 \mathrm{hrs}$ p.i. Briefly, the total RNA was treated with alkaline phosphatase and tobacco acid pyrophosphatase in turn. The decapped mRNA was ligated with a 5'-RACE adaptor supplied with the kit. First strand cDNA was synthesized with a Ha127-specific primer RT: 5'-GACGTGGTCGCCGTTGAGAAAC-3', followed by two rounds of nested PCR with primer pairs, 5'-RACE outer primer/ GSP1: 5'-ATCATACGCCACCAGCTAGCC-3' and 5'-RACE inner primer/GSP2: 5'-CGACAAACACTGCGTCTCT-3'. The final produced $\mathrm{PCR}$ products were gel-purified, inserted into pGEM $^{\circledR}-\mathrm{T}$ Easy vector and sequenced with $\mathrm{T} 7$ primer.

Western blot analysis of Ha127 protein. Infected cells were harvested at the designated time points (see above). After being washed in PBS, the cells were lysed in SDS-PAGE sample buffer by boiling for 5 mins. Western blot analysis was performed as described by Du et al. (2006).
Confocal laser scanning microscopy of cells expressing Ha127EGFP fusion protein. To monitor the localization of $\mathrm{Ha} 127$ in HaNPV-infected cells, Ha127 was expressed in frame with EGFP as described (Nie et al., 2006) with some modifications. A donor plasmid was constructed as follows. An XhoI-HindIII fragment harboring the EGFP ORF was amplified with primer pair EGFPF: ACTCGAGATGGTGAGCAAGGGCG, EGFP-R: AAAGCTTT TACTTGTACAGCTCGTC, and pEGFP-C1 as the template. The fragment was inserted into XhoI-HindIII-digested pHaFastBac1 (Wang et al., 2003) to produce pHaFast-EGFP. The Ha127 coding region without termination codon TGA was amplified from HaNPV genome bacmid with primers 127-F and 127R-TGANull: ACTCGAGATGCTCAATCGTGGCGATAG, and cloned into corresponding restriction sites of plasmid pHaFast-EGFP to obtain pHaFast-127EGFP that was used to transform E.coli DH10B cells containing the HaNPV bacmid (HaBacHZ8) and transposition helper plasmid pMON7124 (Wang et al., 2003). The obtained recombinant HaNPV bacmid was named vHa-Ha127-EGFP. The control recombinant bacmid was constructed in the same fashion with the exception of the change in the restriction site of forward primer EGFP-F to BamHI and designated as vHa-EGFP.

HzAM1 cells were infected with vHa-Ha127-EGFP or vHaEGFP. At 72 hrs p.i., cells were examined under a Leica confocal laser scanning microscopy for sub-cellular localization analysis.

Immunofluorescence microscopy of cells expressing Ha127. Monolayer of HzAM1 cells were grown in a plastic Petri dish and infected with BVs of HaNPV. At 48 hrs p.i., cells were incubated with the prepared polyclonal Ha127 antiserum, followed by the incubation with FITC-conjugated goat anti-rabbit IgG and nucleus-specific stain DAPI (Sigma) for $1 \mathrm{hr}$ (An et al., 2005). The infected cells incubated with pre-immune serum were used as a negative control. The stained cells were examined under Leica confocal laser microscope.

\section{Results}

\section{Sequence analysis of Hal27 gene}

The Ha127 amino acid sequence was predicted one N-glycosylation site (aa 7-10), one casein kinase II phosphorylation site (aa 27-30) and seven protein kinase $C$ phosphorylation sites (aa 8-10, 27-29, 69-71, 88-90, 107-109, 110-112, 130-132) by using the MotifScan tool on the website http:// myhits.isb-sib.ch/cgi-bin/motif_scan. In addition, a potential leucine zipper motif was observed at aa 34-55.

Search in the databases revealed that the Ha127 is a rare protein whose homologies were found only in some members of Alphabaculovirus including HzSNPV ORF131 (Chen et al., 2002), TnSNPV ORF135 (Willis et al., 2005), OrleNPV ORF129 (GenBank, NC_010276), ChchNPV ORF142 (van Oers et al., 2005), MacoNPV (B) ORF35 (Li et al., 2002a), LdMNPV ORF127 (Kuzio et al., 1999), SpltNPV ORF120 (Pang et al., 2001), ClbiNPV ORF126 (Zhu et al., 2009), HaMNPV ORF34 (GenBank NC_011615), MacoNPV (A) ORF40 (Li et al., 2002b), SpliNPV ORF3 (EMBL Acc. AJ003131.1), and SeMNPV ORF28 (IJkel et al., 1999). The alignment of aa 


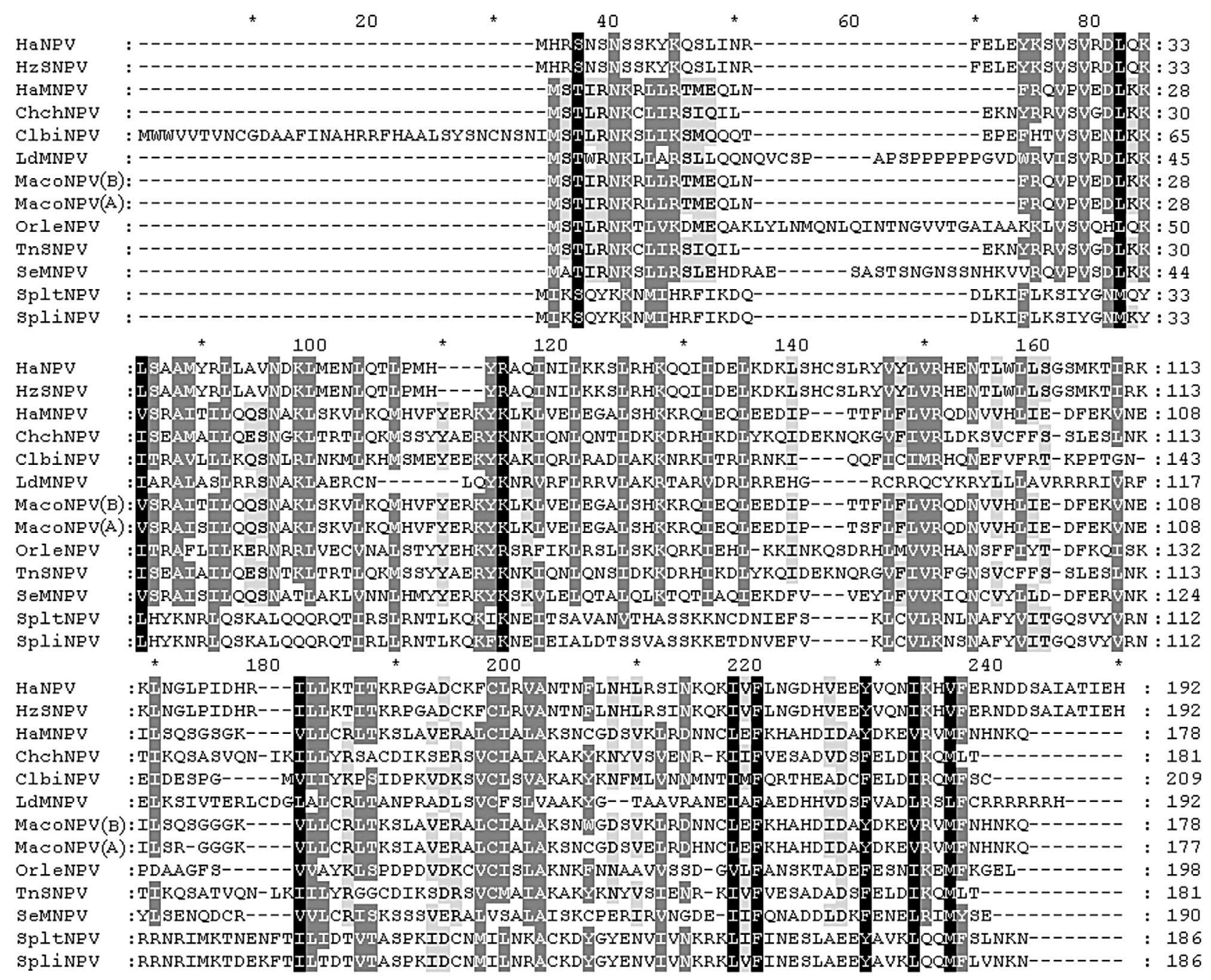

Fig. 1

Amino acid sequence alignment of HaNPV Ha127 and its homologs

Black shading shows $100 \%$ identity and grey $80 \%$ identity.

sequences indicated only one completely conserved aa residue in those homologies. The protein Ha127 had the highest identity (100\%) with HzSNPV ORF131 and lower sequence identity with the other homologues ranging from $22 \%$ to $28 \%$ (Fig. 1).

Also, to determine the 5'-end of Ha127 transcript, 5'-RACE analysis was performed with total RNA isolated at 18 and 48 hrs p.i. After two rounds of nested PCR, a single band was visualized on agarose gel (data not shown). Four clones were sequenced and the transcription initiation site of Ha127 gene was 188 nts upstream of the translation initiation codon ATG and positioned at the A base (Fig. 2).

\section{TTTTGGTAATGACTCATTTGGGTCAGGCTATTOGGCGGTTGGTTGTTATTTAATACTGTA $\rightarrow$ Transcription start site 120361 AOGACTTATACCATGCATCGTTOCAATAGTAACAGCAGCAAATACAAACAATOGCTGATA}

Fig. 2

Determination of transcription initiation site in Ha127 transcript by 5'-RACE analysis

ATG - translation initiation codon; numbers at the left side - location of the sequence in HaNPV G4 genome. 


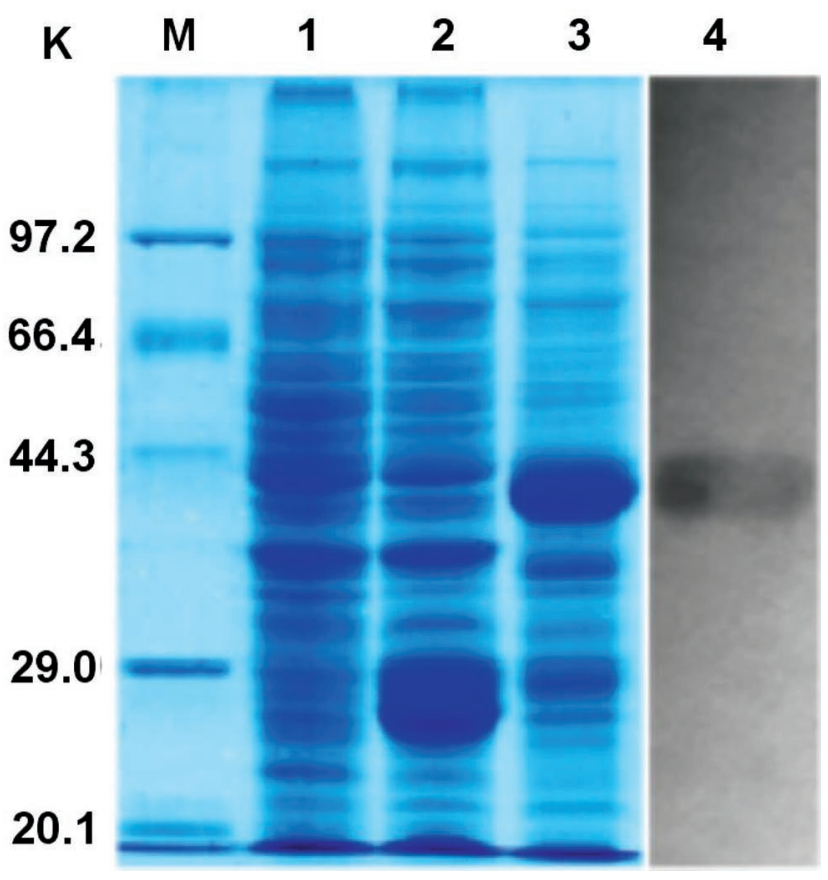

Fig. 3

Expression of GST-Ha127 fusion protein in $E$. coli detected by SDS-PAGE $(1,2,3)$ and Western blot $(4)$

Protein size marker (M), no-plasmid transformed E. coli (1), E. coli transformed with empty plasmid (2), E. coli transformed with the plasmid containing GST-Ha127 fusion gene $(3,4)$

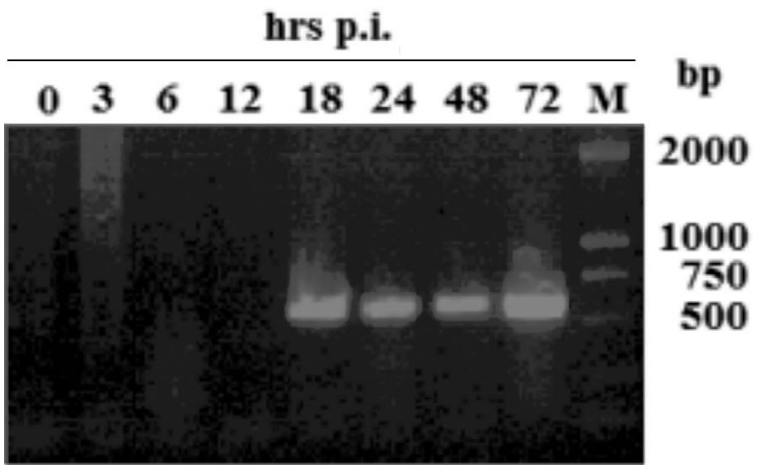

Fig. 4

Detection of HaNPV Ha127 transcripts in HaNPV-infected HzAM1 cells by RT-PCR

DNA size marker (M).

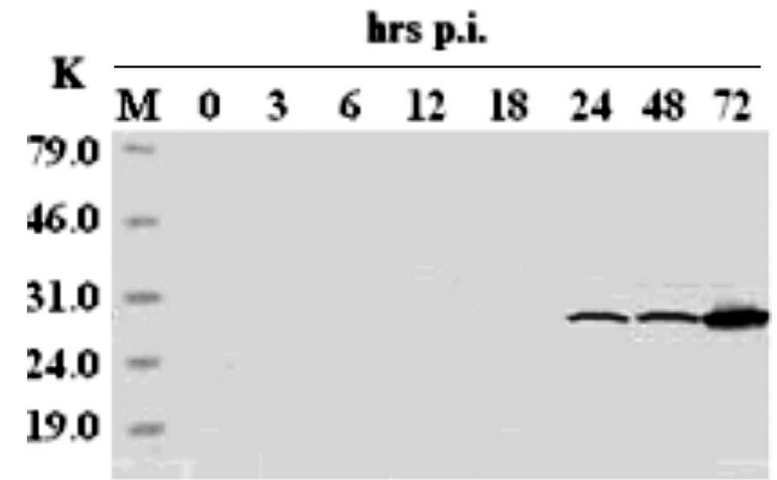

Fig. 5

Detection of HaNPV Ha127 in HaNPV-infected HzAM1 cells by Western blot analysis

Protein size marker (M)

(a)
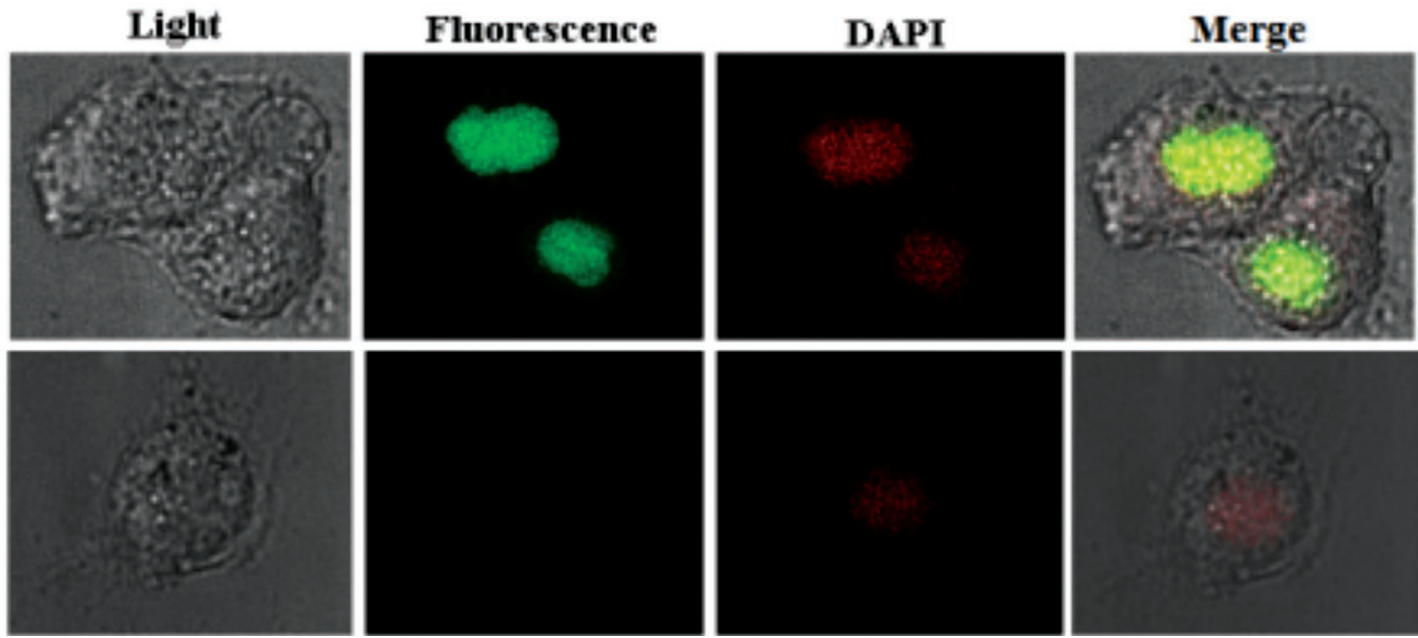

Fig. 6

Sub-cellular localization of Ha127 in HaNPV-infected HzAM1 cells revealed by immunofluorescence Cells incubated with Ha127 antiserum (a) and pre-immune serum (b) at 48 hrs p.i. 


\section{Expression of GST-Ha127 fusion protein in E. coli}

In order to express Ha127 in vitro, a vector pGEX-GSTha127 was constructed and introduced into E. coli BL21 (DE3) LysS cells. After being induced and analyzed by SDS-PAGE, an intensive protein band was detected. Western blot analysis using GST antibody further confirmed that this band was the fusion protein GST-Ha127 (Fig. 3). The size of GST-Ha127 was about $40 \mathrm{~K}$, smaller than theoretical size of $48.6 \mathrm{~K}$ suggesting that Ha127 protein was not expressed completely. Yet, the fusion protein was purified and used to immunize rabbits for preparation of the antiserum.

\section{Expression of Hal27 transcripts in HaNPV-infected HzAM1 cells}

The transcription of Ha127 was examined by RT-PCR analysis using total RNA isolated from HaNPV-infected HzAM1 cells at different time points p.i. A single Ha127 transcript was detected at $18 \mathrm{hrs}$ p.i. and remained detectable until $72 \mathrm{hrs}$ p.i. suggesting that Ha127 was a late gene (Fig. 4).

To investigate the time course of Ha127 expression, the total proteins of HaNPV-infected cells were sampled and analyzed by Western blot analysis with Ha127 antiserum (Fig. 5). The results revealed that a protein band of approx. $28 \mathrm{~K}$ showed a strong reaction. The protein was detectable at $24 \mathrm{hrs} \mathrm{p.i.} \mathrm{and} \mathrm{its} \mathrm{amount} \mathrm{increased} \mathrm{till} 72 \mathrm{hrs}$ p.i. The size of the immunoreactive protein was larger than the predicted Ha127 gene product, indicating that this protein might be a subject to post-translational modification.

\section{Sub-cellular localization of Ha127}

The sub-cellular localization of Ha127 protein was investigated by the expression of fusion protein Ha127-EGFP in HzAM1 cells using HaNPV Bac-to-Bac system. It seemed that the fusion protein Ha127-EGFP was localized in the nucleus (data not shown). However, the expression of Ha127-EGFP was promoted by the HaNPV polyhedrin promoter, a very late one in the baculovirus genome to promote the transcription of polyhedrin. So, to confirm further the localization of Ha127 protein, an immunofluorescence assay using GST-Ha127 antiserum and DAPI staining was performed. The results revealed that the fluorescence signal was strictly concentrated within the nucleoplasm and no fluorescence was detected in the cytoplasm. The HaNPV-infected cells used as negative control incubated with a pre-immune serum and FITC-conjugated goat anti-rabbit IgG did not show a detectable fluorescence signal (Fig. 6).

\section{Discussion}

In the present study we described some preliminary characteristics of Ha127 gene and the corresponding protein. Based on the comparison of baculovirus genomes, 62 genes were conserved and believed as the core genes for all lepidopteran nucleopolyhedroviruses (Jehle et al., 2006). Among the sequenced baculovirus genomes up to the present, Ha127 is a rare gene whose homologies were observed only in some members of the genus Alphabaculovirus, which implied that it might be responsible for a specific role related to its host.

To characterize Ha127 protein, its expression and subcellular localization were analyzed. Results obtained by RT-PCR and Western blot analysis suggested that Ha127 was a late gene. Fusion expression with EGFP and immunofluorescence analysis showed that Ha127 protein was localized in the nucleus.

The gene expression pattern in the baculovirus life cycle is regulated by a temporal cascade mode. Based on this regulation fashion, the gene expression of baculovirus can be divided into 4 phases: immediate-early, delayed-early, late, and very late. After infection, the immediate-early and delayed-early genes are transcribed by the host RNA polymerase II and most of them are thought to be involved in viral DNA replication and late gene expression (Todd et al., 1996). Expression of delayed-early genes, however, appears to be dependent on the presence of immediate-early gene products (Kelly et al., 2007). Late and very-late genes are transcribed by the viral RNA polymerase, whose expression is regulated by the early genes. Late gene expression is dependent on the replication of virus DNA and late proteins are frequently involved in the regulation of late and very late gene expression (Hefferon, 2004). Very late protein expression is characterized by the prominent synthesis of p10 and polyhedrin. These proteins are often involved in the processes of occlusion and cell lysis (Hasnain et al., 1997). The results indicated that Ha127 gene might be associated with the late or very late gene expression.

In this paper some basic characteristics of Ha127 gene were studied, but many attributes about this gene remained unidentified. To further understand the function of Ha127 gene, certain deletion and mutagenesis studies using a HaNPV bacmid would be necessary.

Acknowledgements. The authors thank Dr. Hua Ling Wang, State Key Laboratory of Virology, Wuhan Institute of Virology, Chinese Academy of Sciences, Wuhan, for providing HaNPV Bac-to-Bac system. This study was supported by the grant 05JDG048 from the High Talent Fund Grant of Jiangsu University and by the grant 07KJB180013 from the College Natural Science Foundation of Jiangsu Province.

\section{References}

An SH, Wang D, Zhang-Nv Y, Guo ZJ, Xu HJ, Sun JX, Zhang CX (2005): Characterization of a late expression gene, open reading frame 128 of Helicoverpa armigera single 
nucleocapsid nucleopolyhedrovirus. Arch. Virol. 150, 2453-2466. doi:10.1007/s00705-005-0605-y

Chen X, IJkel WFJ, Tarchini R, Sun X, Sandbrink H, Wang H, Peters S, Zuidema D, Lankhorst RK, Vlak JM, Hu Z (2001): The sequence of the Helicoverpa armigera single nucleocapsid nucleopolyhedrovirus genome. J. Gen. Virol. 82, 241-257.

Chen X, Zhang W-J, Wong J, Chun G, Lu A, McCutchen BF, Presnail JK, Herrmann R, Dolan M, Tingey S, Hu ZH, Vlak JM (2002): Comparative analysis of the complete genome sequences of Helicoverpa zea and Helicoverpa armigera single-nucleocapsid nucleopolyhedroviruses. J. Gen. Virol. 83, 673-684.

Du MF, Yin XM, Guo ZJ, Zhu LJ (2006): Characterization of a late gene, ORF60 from Bombyx mori nucleopolyhedrovirus. J. Biochem. Mol. Biol. 39, 737-742.

Guo ZJ, An SH, Wang D, Liu YH, Kumar VS, Zhang CX (2005): Characterization of $\mathrm{Ha} 29$, a specific gene for Helicoverpa armigera single-nucleocapsid nucleopolyhedrovirus. J. Biochem. Mol. Biol. 38, 354-359.

Hasnain SE, Jain A, Habib S, Ghosh S, Chatterji U, Ramachandran A, Das P, Venkaiah B, Pandey S, Liang B, Ranjan A, Natarajan K, Azim CA (1997): Involvement of host factors in transcription from baculovirus very late promoters - a review. Gene 190, 113-118. doi:10.1016/S03781119(96)00827-X

Hefferon KL (2004): Baculovirus late expression factors. J. Mol. Microbiol. Biotechnol. 7, 89-101. doi:10.1159/ $\underline{000078652}$

IJkel WFJ, van Strien EA, Heldens JGM, Broer R, Zuidema D, Goldbach RW, Vlak JM (1999): Sequence and organization of the Spodoptera exigua multicapsid nucleopolyhedrovirus genome. J. Gen. Virol. 80, 3289-3304.

Jehle JA, Blissard GW, Bonning BC, Cory JS, Herniou EA, Rohrmann GF, Theilmann DA, Thiem SM, Vlak JM (2006): On the classification and nomenclature of baculoviruses: a proposal for revision. Arch. Virol. 151, 1257-1266. doi:10.1007/s00705-006-0763-6

Kelly BJ, King LA, Possee RD (2007): Introduction to baculovirus molecular biology. In Murhammer DW (Ed.): Baculovirus and Insect Cell Expression Protocols. 2nd ed., Vol. 338. Humana Press, Totowa, New Jersey, pp. 25-53.

King ABS (1994): Heliothis/Helicoverpa armigera (Lepidoptera: Noctuidae). In Matthews G, Tunstall J (Eds): Insect Pests of Cotton. CAB International, Wallingford, UK, pp. 445-467.

Kuzio J, Pearson MN, Harwood SH, Funk CJ, Evans JT, Slavicek JM, Rohrmann GF (1999): Sequence and analysis of the genome of a baculovirus pathogenic for Lymantria dispar. Virology 253, 17-34. doi:10.1006/viro.1998.9469

Li L, Donly C, Li Q, Willis LG, Keddie BA, Erlandson MA, Theilmann DA (2002a): Identification and genomic analysis of a second species of nucleopolyhedrovirus isolated from Mamestra configurata. Virology 297, 226-244. doi:10.1006/viro.2002.1411

Li L, Li Q, Willis LG, Erlandson M, Theilmann DA, Donly C (2002b): Complete comparative genomic analysis of two field isolates of Mamestra configurata nucleopoly-
hedrovirus-A. J. Gen. Virol. 86, 91-105. doi:10.1099/ vir.0.80488-0

Long G, Westenberg M, Wang HL, Vlak JM, Hu ZH (2006): Function, oligomerization and N-linked glycosylation of the Helicoverpa armigera single nucleopolyhedrovirus envelope fusion protein. J. Gen. Virol. 87, 839-846. doi: 10.1099/vir.0.81592-0

Nie Y, Wang Q, Liang C, Fang M, Yu Z, Chen X (2006): Characterization of ORF2 and its encoded protein of the Helicoverpa armigera nucleopolyhedrovirus. Virus Res. 116, 129-135. doi:10.1016/j.virusres.2005.09.007

Pan XY, Long G, Wang RR, Hou SW, Wang HY, Zheng YT, Sun XL, Westenberg M, Deng F, Wang HL, Vlak JM, Hu ZH (2007): Deletion of a Helicoverpa armigera nucleopolyhedrovirus gene encoding a virion structural protein (ORF107) increases the budded virion titre and reduces in vivo infectivity. J. Gen. Virol. 88, 3307-3316. doi:10.1099/vir.0.83363-0

Pang Y, Yu J, Wang L, Hu X, Bao W, Li G, Chen C, Han H, Hu S, Yang H (2001): Sequence analysis of the Spodoptera litura multicapsid nucleopolyhedrovirus genome. Virology 287, 391-404. doi:10.1006/viro.2001.1056

Song JJ, Wang RR, Deng F, Wang HL, Hu ZH (2008): Functional studies of per os infectivity factors of Helicoverpa armigera single nucleocapsid nucleopolyhedrovirus. J. Gen. Virol. 89, 2331-2338. doi:10.1099/vir.0.2008/002352-0

Theilmann DA, Blissard GW, Bonning B, Jehle JA, O'Reilly DR, Rohrmann GF, Thiem S, Vlak JM (2005): Baculoviridae. In Fauquet CM, Mayo MA, Maniloff J, Desselberger U, Ball LA (Eds): Virus Taxonomy: The Eighth Report of the International Committee on Taxonomy of Viruses. Elsevier Academic Press, New York, NY, pp. 177-185.

Todd JW, Passarelli AL, Lu A, Miller LK (1996): Factors regulating baculovirus late and very late gene expression in transient-expression assays. J. Virol. 70, 2307-2317.

van Oers MM, Abma-Henkens MH, Herniou EA, de Groot JC, Peters S, Vlak JM (2005): Genome sequence of Chrysodeixis chalcites nucleopolyhedrovirus, a baculovirus with two DNA photolyase genes. J. Gen. Virol. 86, 2069-2080. doi:10.1099/vir.0.80964-0

Wang D, An SH, Guo ZJ, Xu HJ, Zhang CX (2005): Characterization of Helicoverpa armigera nucleopolyhedrovirus orf33 that encodes a novel budded virion derived protein, BVe31. Arch. Virol. 150, 1505-1515. doi:10.1007/s00705005-0534-9

Wang H, Deng F, Pijlman GP, Chen X, Sun X, Vlak JM, Hu Z (2003): Cloning of biologically active genomes from a Helicoverpa armigera single-nucleocapsid nucleopolyhedrovirus isolate by using a bacterial artificial chromosome. Virus Res. 97, 57-63. doi:10.1016/j.virusres.2003.07.001

Wang H, Wu D, Deng F, Peng H, Chen X, Lauzon H, Arif BM, Jehle JA, Hu Z (2004): Characterization and phylogenetic analysis of the chitinase gene from the Helicoverpa armigera single nucleocapsid nucleopolyhedrovirus. Virus Res. 100, 179-189. doi:10.1016/j.virusres.2003.11.015

Wang Q, Liang CY, Song JH, Chen XW (2007): HA2 from the Helicoverpa armigera nucleopolyhedrovirus: a WASPrelated protein that activates Arp2/3-induced actin 
filament formation. Virus Res. 127, 81-87. doi:10.1016/ j.virusres.2007.03.021

Wang Q, Wang Y, Liang CY, Song JH, Chen XW (2008): Identification of a hydrophobic domain of HA2 essential to morphogenesis of Helicoverpa armigera nucleopolyhedrovirus. J. Virol. 82, 4072-4081. doi:10.1128/JVI.02319-07

Willis LG, Siepp R, Stewart TM, Erlandson MA, Theilmann DA (2005): Sequence analysis of the complete genome of Trichoplusia ni single nucleopolyhedrovirus and the identification of a baculoviral photolyase gene. Virology 338, 209-226. doi:10.1016/j.virol.2005.04.041

Wu D, Deng F, Sun XL, Wang HL, Yuan L, Vlak JM, Hu ZH (2005): Functional analysis of FP25K of Helicoverpa armigera single nucleocapsid nucleopolyhedrovirus. J. Gen. Virol. 86, 2439-2444. doi:10.1099/vir.0.81110-0 Zhang CX, Ma XC, Guo ZJ (2005): Comparison of the complete genome sequences between $\mathrm{C} 1$ and G4 isolates of the Helicoverpa armigera single nucleocapsid nucleopolyhedrovirus. Virology 333, 190-199. doi:10.1016/ j.virol.2004.12.028

Zhu SY, Yi JP, Shen WD, Wang LQ, He HG, Wang Y, Li B, Wang WB (2009): Genomic sequence, organization and characteristics of a new nucleopolyhedrovirus isolated from Clanis bilineata larva. BMC Genomics 10, 91-99. doi:10.1186/1471-2164-10-91 